\title{
Loss of AA13 LPMOs impairs degradation of resistant starch and reduces the growth of Aspergillus nidulans
}

\author{
Majid Haddad Momeni, Maria Louise Leth, Claus Sternberg, Erwin Schoof, Maike Wennekers Nielsen, \\ Jesper Holck, Christopher T. Workman, Jakob Blæsbjerg Hoof and Maher Abou Hachem* (10)
}

\begin{abstract}
Background: Lytic polysaccharide monooxygenases (LPMOs) are often studied in simple models involving activity measurements of a single LPMO or a blend thereof with hydrolytic enzymes towards an insoluble substrate. However, the contribution of LPMOs to polysaccharide breakdown in complex cocktails of hydrolytic and oxidative enzymes, similar to fungal secretomes, remains elusive. Typically, two starch-specific AA13 LPMOs are encoded by mainly Ascomycota genomes. Here, we investigate the impact of LPMO loss on the growth and degradation of starches of varying resistance to amylolytic hydrolases by Aspergillus nidulans.
\end{abstract}

Results: Deletion of the genes encoding AnAA13A that possesses a CBM20 starch-binding module, AnAA13B (lacking a CBM20) or both AA13 genes resulted in reduced growth on solid media with resistant, but not soluble processed potato starch. Larger size and amount of residual starch granules were observed for the AA13-deficient strains as compared to the reference and the impairment of starch degradation was more severe for the strain lacking AnAA13A based on a microscopic analysis. After 5 days of growth on raw potato starch in liquid media, the mount of residual starch was about fivefold higher for the AA13 gene deletion strains compared to the reference, which underscores the importance of LPMOs for degradation of especially resistant starches. Proteomic analyses revealed substantial changes in the secretomes of the double AA13 gene deletion, followed by the AnAA13A-deficient strain, whereas only a single protein was significantly different in the proteome of the AnAA13B-deficient strain as compared to the reference.

Conclusions: This study shows that the loss of AA13, especially the starch-binding AnAA13A, impairs degradation of resistant potato starch, but has limited impact on less-resistant wheat starch and no impact on processed solubilized starch. The effects of LPMO loss are more pronounced at the later stages of fungal growth, likely due to the accumulation of the less-accessible regions of the substrate. The striking impairment in granular starch degradation due to the loss of a single LPMO from the secretome offers insight into the crucial role played by AA13 in the breakdown of resistant starch and presents a methodological framework to analyse the contribution of distinct LPMOs towards semi-crystalline polysaccharides under in vivo conditions.

Keywords: Ascomycota, Aspergillus nidulans, Biomass, Biorefinery, Carbohydrate oxidation, Functional genomics, Recalcitrant polysaccharides

*Correspondence: maha@bio.dtu.dk

Department of Biotechnology and Biomedicine, Technical University

of Denmark, Søltofts Plads, 2800 Kgs. Lyngby, Denmark

\section{Background}

The polysaccharide polymers cellulose (produced at $10^{11}$ t per annum) [1], chitin (similar production to cellulose) and starch (produced at $10^{9} \mathrm{t}$ per annum) [2] are the most 
abundant renewable biomass resources on the globe. Fungal secretomes contain numerous extracellular enzymes targeting complex polysaccharides, e.g. lignocellulose and starch [3], both organized as insoluble semi-crystalline composites. Hydrolytic breakdown of the crystalline regions in these polymers requires the sequestering of single glycan chains from the semi-crystalline polymer matrix, which poses a large energetic penalty and lowers the efficiency of hydrolytic enzymes. Lytic polysaccharide monooxygenases (LPMOs) are copper-dependent oxidoreductases discovered in 2010 [4]. Uniquely, LPMOs catalyse the oxidative cleavage of glycosidic linkages in the crystalline regions of chitin, cellulose and starch using an exogenous reductant and $\mathrm{H}_{2} \mathrm{O}_{2}$ as the preferred co-substrate [5, 6]. Notably, LPMOs display synergy with hydrolytic enzymes in the degradation of chitin [4], cellulose [7] and starch [8, 9]. Currently, LPMOs are recognized as key players in recalcitrant biomass degradation and are considered as integral components in commercial enzyme cocktails targeting crystalline polysaccharides. Despite the significant industrial interest in LPMOs and recent advances in understanding their structures, mechanism and substrate-interactions [10, 11], insight is still lacking into the impact of these powerful oxidoreductases during fungal growth on semi-crystalline polysaccharides. Most functional studies on LPMOs employ a single LPMO, occasionally in the presence or absence of a hydrolase targeting the same substrate. Additionally, secretomes from distinct fungi have been shown to boost the efficiency of degradation of lignocellulose [11]. However, the contribution of individual LPMOs is not readily measurable due to the multiplicity and diversity of secreted LPMOs, other oxidoreductases and glycoside hydrolases (GHs) during growth on complex biomass polysaccharides $[12,13]$.

Starch is a composite of the mainly linear amylose, consisting of $\alpha-(1,4)$-linked glucosyl units and the major component amylopectin, constituting up to $70-80 \%$ per weight in most plants. Amylopectin consists of $\alpha-(1,4)$ linked linear glucosyl chains and 5-6\% $\alpha$-1,6-glucosidic branch points $[14,15]$. In planta, starch is synthesized as granules that vary in size, morphology and crystallinity (15-45\%). In both amylose and amylopectin, the formation of double helices by linear $\alpha$-glucan chains promotes their packing into crystalline lamellae that, in amylopectin, alternate radially with amorphous regions. The $\mathrm{A}$ and $\mathrm{B}$ allomorphs are the most commonly observed in cereal and tuber starches, respectively [12]. The resistance of starch to degradation by hydrolases is strongly correlated to the details of the $\alpha$-glucan packing in the granule and the crystal allomorphs. Thus, cereal starches are considered susceptible, whereas unprocessed tuber starches are considered resistant $[13,14]$.
Currently, starch-active LPMOs are classified into auxiliary activity family 13 (AA13) in the CAZy database [16]. To date, AA13 LPMOs are encoded by the genomes of mainly saprotrophs and plant pathogens from Ascomycota. Frequently, but not invariantly, two AA13s are encoded in a single genome: a catalytic module (CM) and a modular enzyme comprising an $\mathrm{N}$-terminal $\mathrm{CM}$ appended to a C-terminal starch-binding module of family 20 (CBM20) via a linker region (Fig. 1) [17]. We have previously shown that $A n \mathrm{AA} 13 \mathrm{~B}$ was ranked among the top 10 upregulated proteins in the Aspergillus nidulans secretomes during growth on three different starches, while the modular AnAA13A that is appended to a CBM20 was the most abundant in the starch-binding secretome fraction [13]. This striking abundance in the starch-induced secretomes, suggested that AA13s may play a pivotal role in starch deconstruction. The chemical simplicity of starch and presence of only two AA13s offer an appropriate model to investigate the impact of LPMOs on the deconstruction of semi-crystalline polysaccharides in vivo.

In this study, we have generated two single and a double knockout (KO) of the AA13s genes in A. nidulans and analysed the impact of these gene deletions on the growth and starch utilization by $A$. nidulans. The loss of AA13s, especially the enzyme with the CBM20, had a major impact on the ability of the fungus to degrade resistant potato starch. By contrast, AA13s appeared less crucial for the degradation of the less-resistant wheat starch and soluble processed potato starch. Altogether, these findings underscore the instrumental role of LPMOs, especially those appended to CBMs, in fungal breakdown of granular resistant starch and establish

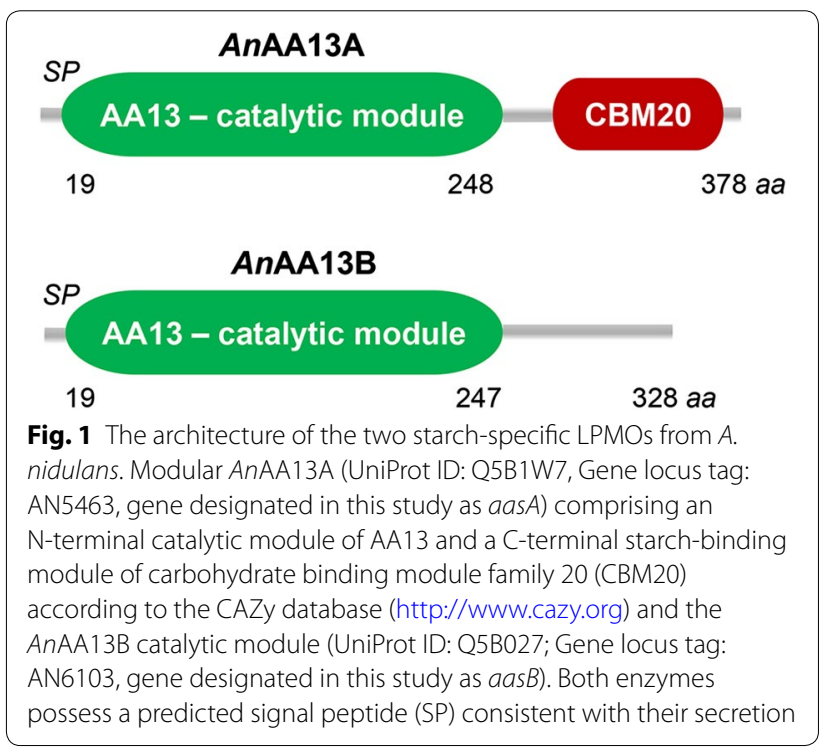


a framework to dissect the contribution of LPMOs in breakdown of recalcitrant polysaccharides.

\section{Results}

Construction of gene-deletion mutants of the starch-specific AA13 from A. nidulans

Aspergillus nidulans encodes two LPMOs of AA13 sharing $83.8 \%$ primary structure identity in their catalytic modules: AnAA13A (AN5463, the gene is henceforth designated aas $A$, auxiliary activity family 13 specific for starch), which is the modular enzyme harbouring a C-terminal starch-binding CBM20 and AnAA13B (AN6103, gene designated as aasB) (Fig. 1). To explore the significance of these LPMOs for growth on starch, a double and two individual single gene-deletion strains of the encoding aas $A$ and $a a s B$ genes were generated. The up- and downstream sequences flanking each of the two LPMO genes were incorporated into the USER-compatible vector backbone pU2002-A containing AfpyrG encoding orotidine-5'-phosphate decarboxylase from Aspergillus fumigatus as selection marker, under the control of its native promoter and terminator flanked by a direct repeat (DR) [18]. This procedure generated the gene-deletion vectors pU2000-aas A and pU2000aas $B$, which were digested to yield linear gene-targeting substrates (GTSs). The non-homologous end-joiningdeficient $A$. nidulans NID1 strain [18, 19], used as a reference strain, was transformed individually with each GTS (Additional file 1: Table S1). The aas $A \Delta$ and aas $B \Delta$ deletion mutants were identified as homokaryotic deletions by diagnostic tissue-PCR, respectively, following the mutant validation scheme in Additional file 1: Fig. S1 and Table S2. To assess the effect of losing both AA13 LPMOs and their functional redundancy, we re-streaked spores of aasB $\Delta:: A f p y r G$ on 5-FOA supplemented medium to select for the loss of the pyrG marker hereby generating a uracil and uridine auxotrophic aas $B \Delta$ strain (Additional file 1: Fig. S1). Protoplasts were generated from this aas $B \triangle$ strain to create the aas $A \triangle$ aas $B \Delta$ double-deletion strain by employing the aas $A$ GTS. The resulting transformants were re-streaked and analysed by diagnostic tissue-PCR to identify the mutant strains (Additional file 1: Fig. S1). Restoration of the $\arg B 2$ allele to functional $\arg B$ was carried out using CRISPR/Cas9 and oligo-nucleotide-mediated gene-editing [15]. A single prototrophic strain for arginine representing each of the single and double deletions was chosen for further analyses.

\section{Deletion of AA13 LPMO genes impairs growth and degradation of especially resistant starch by $A$. nidulans}

To evaluate the impact of the AA13s, we used three different starches: (1) raw granular potato starch, (2) wheat granular starch and (3) processed soluble potato starch. The raw potato starch comprises large (up to $100 \mu \mathrm{m}$ in diameter) granules of the tightly packed B-type allomorph, considered as a resistant starch type, as compared to the A-type allomorph of the smaller cereal (including wheat) granules that are considered more susceptible to amylolytic hydrolases $[13,14,16]$. The processed potato starch is a soluble hydrated form (granular structure is disrupted), which is highly accessible to $\alpha$-amylases. The $A$. nidulans reference and AA13 gene deletion strains were grown for up to 9 days on minimal media agar plates supplemented with glucose, soluble potato starch, or resistant potato starch, which has been thoroughly washed as previously described [17] to minimize the presence of soluble easily accessible residues to fungal $\alpha$-glucan hydrolases. The growth phenotype of the reference and AA13 gene deletion strains was not distinguishable on glucose (Fig. 2a-d, Additional file 1: Fig. S2). The phenotype of the mutants was also rather similar to the reference strain on soluble potato starch (Fig. 2e-h, Additional file 1: Fig. S3). Strikingly, the strains lacking AA13s showed a clearly distinguishable growth phenotype on resistant potato starch, as compared to the reference (Fig. 2, Supplementary file Fig. S4). Remarkably, this difference was not readily visible during the early growth phase (days 1-5), but became evident from day 6 , as the radial growth rings were smaller and less regular. The (-)AnAA13B strain displayed the least severe phenotype, whereas the growth was most drastically affected for (-)AnAA13A and the double $\mathrm{KO}(-)$ AnAA13A + B (Fig. 2i-l, Additional file 1: Fig. S4).

These results revealed a significant difference in the utilization of resistant starch by the (-)AnAA13 strains, especially those lacking the starch-binding AnAA13A on.

To provide a rationale for the observed phenotypes, we used confocal laser scanning microscopy (CSLM) to analyse growth on the same resistant potato starch. The analysis was performed in a thin agar medium layer supplemented with resistant starch on a microscope slide for $20 \mathrm{~h}$. The relatively short incubation period was set to avoid artefacts related to the drying the thin solidified medium layer and extensive hyphal growth. This analysis revealed that the number and size of granules in the reference strain culture were greatly reduced consistent with efficient degradation of the resistant starch (Fig. 3a, b).

The lack of AnAA13B lowered the efficiency of resistant starch utilization as evident from the larger number and average size distribution of residual starch granules as compared to the reference strain (Fig. $3 \mathrm{a}-\mathrm{c}$ ). However, the least reduction in the size and amount of starch granules, compared to the starting material, was observed for the (-)AnAA13A and the double (-)AnAA13A + B strains 


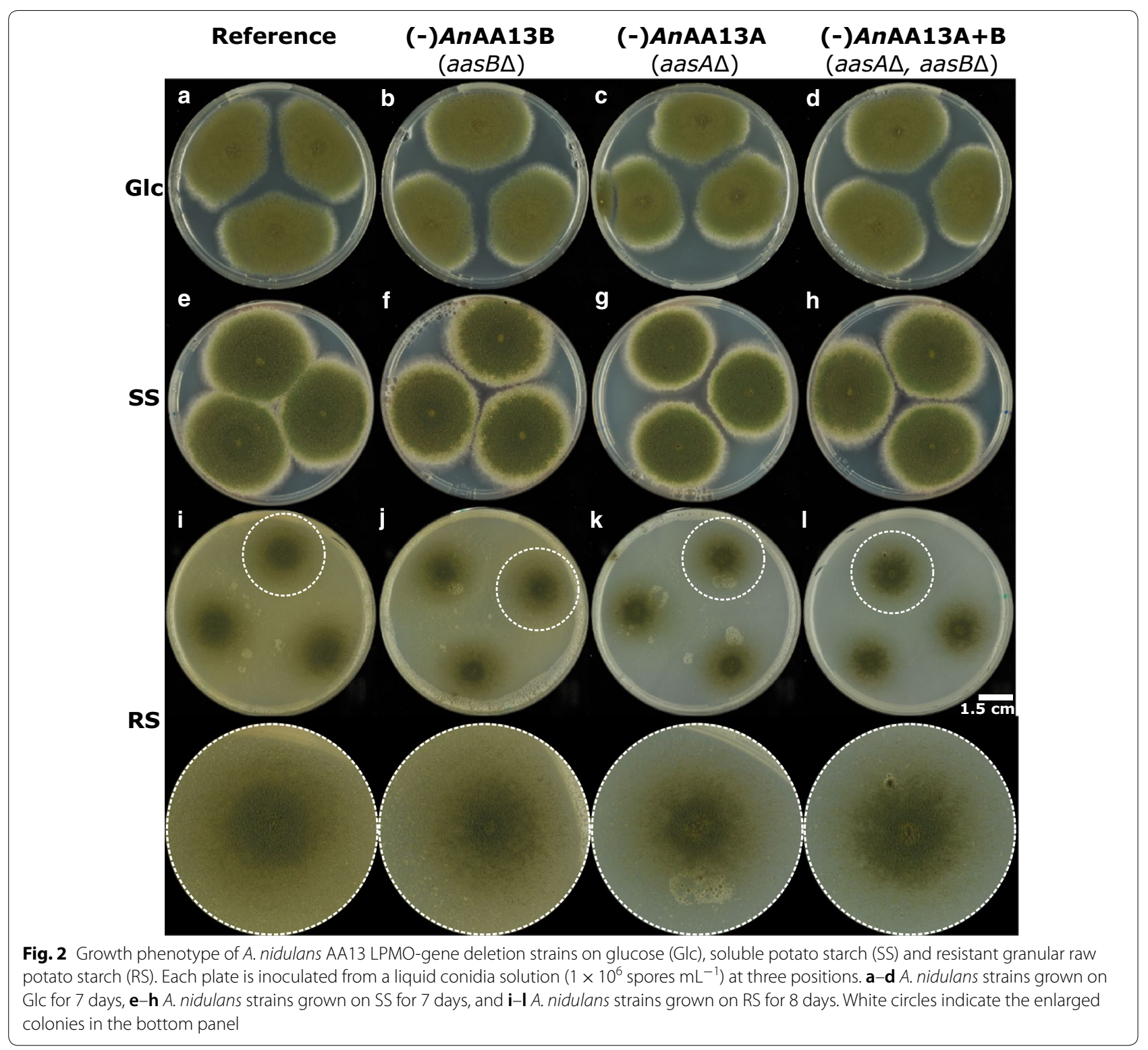

(Fig. 3a, d, e). Thus, the ability of $A$. nidulans to degrade resistant granular starch appeared to be dependent on the presence of the AA13s, especially AnAA13A that harbours the CBM20. To determine the effect of AA13 deficiency on a more susceptible granular starch, a similar analysis including the reference and the (-)AnAA13A $+\mathrm{B}$ strain, was performed on wheat starch, which has been shown to sustain excellent growth and abundant secretion of AA13s by A. nidulans in our previous analysis [18]. Interestingly, the impact of the AA13s deficiency was clearly observable based on the larger number of residual wheat starch granules observed for the (-)AnAA13A + B strain as compared to the reference (Fig. 4). Nonetheless, the impairment in starch degradation was less severe for wheat starch as compared to resistant potato starch based on the marked reduction in the amount and size of granules in the (-)AnAA13A $+\mathrm{B}$ strain, compared to the starting material (Figs 3a, e and 4a, b).

\section{Resistant starch degradation is impaired in liquid cultures of AA13 LPMO gene KOs}

We performed a growth experiment on the same resistant potato starch in liquid media to allow for comparative biochemical and proteomic analyses of the AA13 loss. The culture supernatants of the reference and the gene-deletion strains were analysed for the levels of $\alpha$-amylolytic and $\alpha$-glucosidase activities that mediate the degradation of starch and depolymerization of 

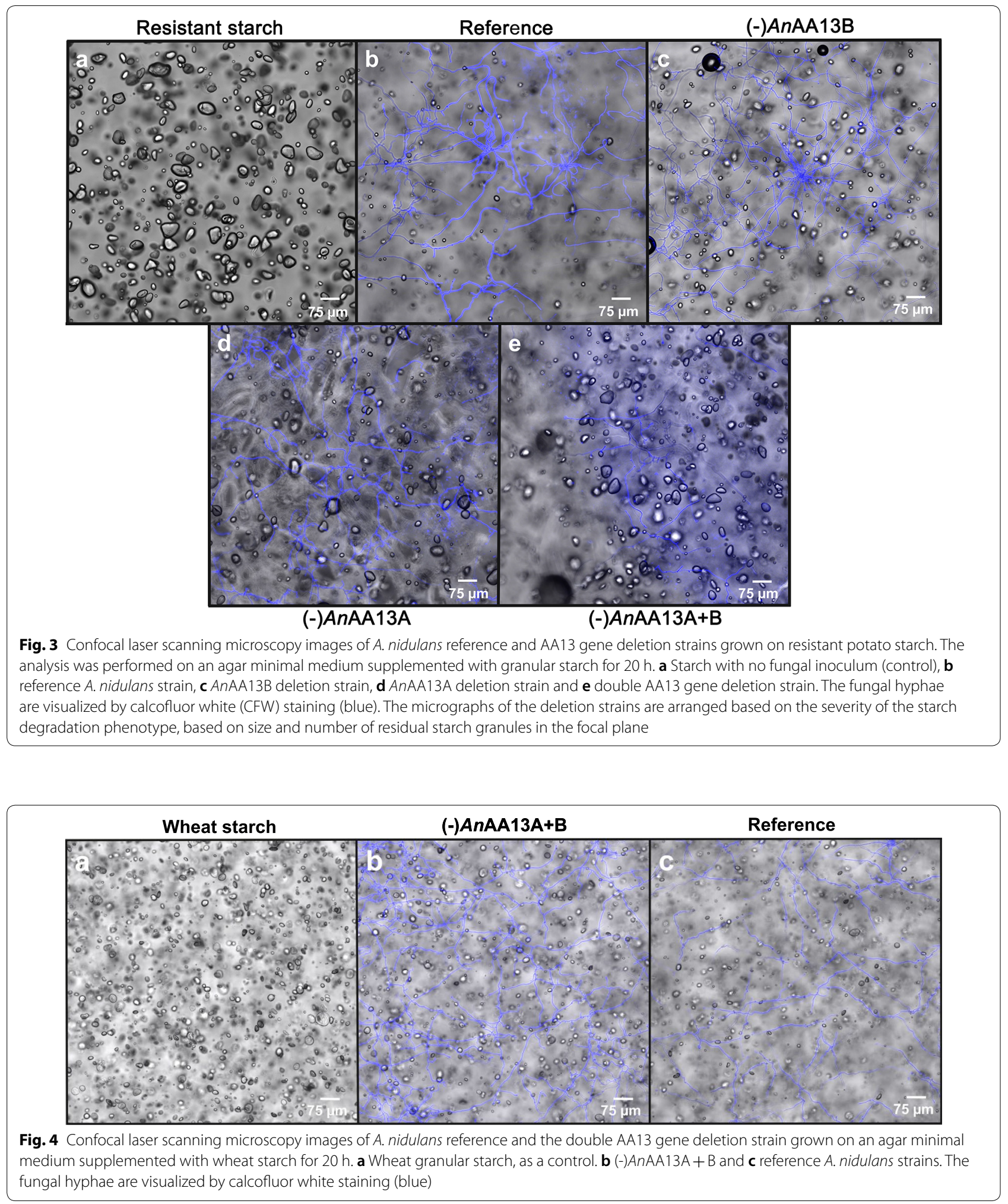

solubilized maltodextrins to glucose, respectively. The levels of these activities were similar, with a trend of modest decrease in the activities of the gene-deletion strains at day 5 (Fig. 5a, b). The protein concentrations in the culture supernatants were also analysed with only modest differences between the reference and 

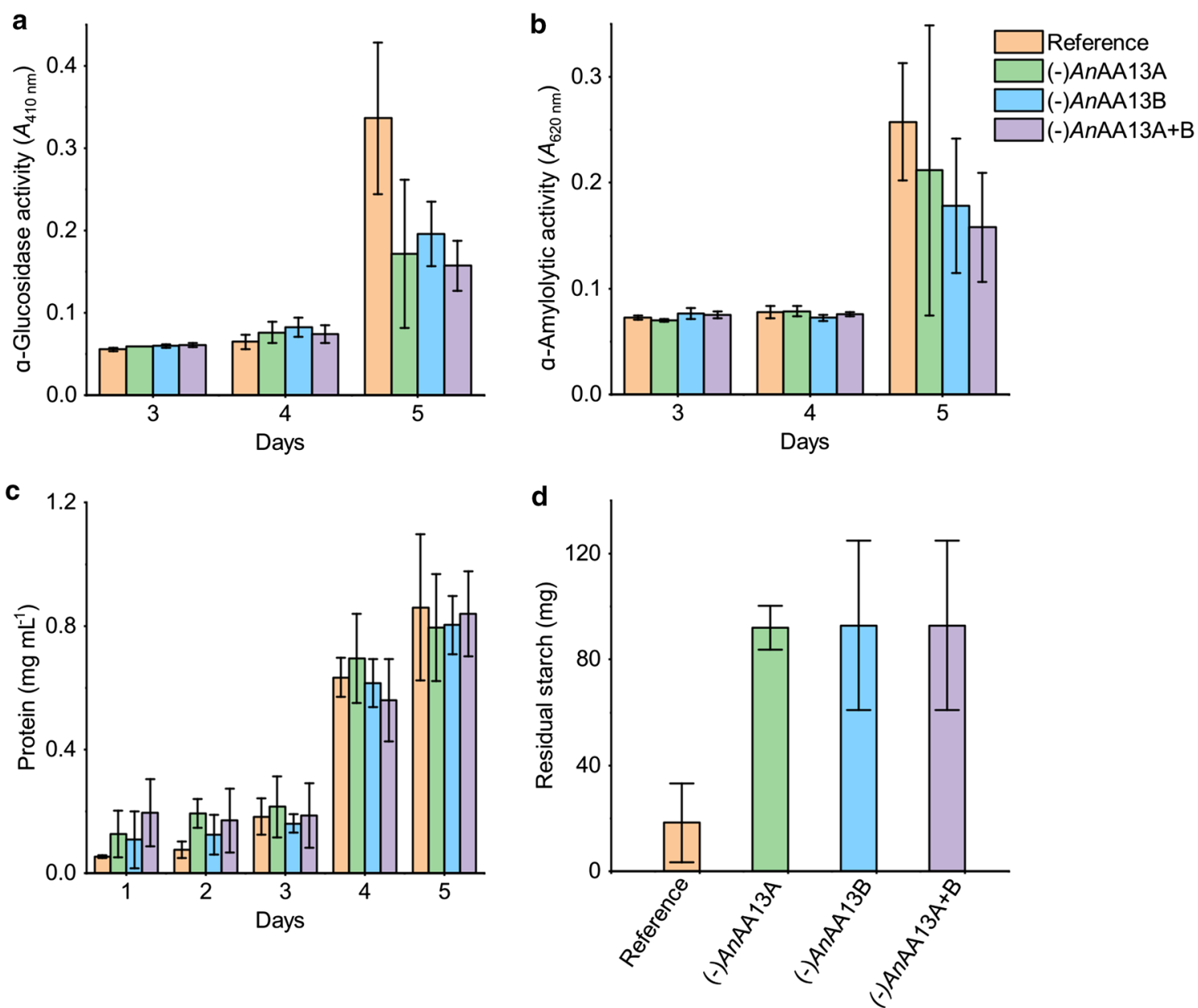

Fig. 5 Analysis of hydrolytic starch-degrading activities, secreted protein levels and residual starch in reference and AA13 gene deletion strains grown in liquid medium on resistant potato starch. $\mathbf{a}$ a-Glucosidase and $\mathbf{b}$ a-amylolytic activities in culture supernatants as analysed using para nitrophenyl a-glucopyranoside and insoluble cross-linked blue starch, respectively. c Protein concentrations in the culture supernatant. $\mathbf{d}$ The residual insoluble starch in the harvested culture suspension (day 5). The start starch concentration in each culture was $0.5 \mathrm{~g}$

AA13 deficient strains (Fig. 5a, b). By contrast, approximately fivefold higher residual starch content was estimated in the AA13 deletion strain cultures compared to the reference strain.

We also compared the biomass in the harvested culture using the mannose content and the wet weight of the retentate form the filtered culture suspension. The cell wall of Aspergillus contains galactomannan [20] as a structural component, which is not present in starch as opposed to glucose, thus it was used as a proxy for fungal biomass formation. Based on these analyses, the changes were modest between the strains, with the direct wet-weight measurement giving a slight reduction of biomass for the gene-deletion strains compared to the reference (Additional file 1: Fig. S5).

\section{Proteomics analysis of the AA13 KOs and reference strains} To bring insight into the impact of the AA13 gene deletion on A. nidulans growth on resistant potato starch, the secretomes of the reference, both the single and the double AA13 gene deletion strains were compared at day 3 and 5 of growth in the liquid culture (Additional file 2). The analysis identified 816 proteins, but strikingly no significant changes of any of these proteins were observed between the reference and AA13 deletion strains at day 3 . Principal component analysis of the data showed that the datasets from the reference and the AA13 deletion strains are clustered together at day 3 , but not on day 5 , especially for the (-)AnAA13A $+\mathrm{B}$ datasets that formed a distinct cluster (Additional file 1: Fig. S6). For (-)AnAA13B, only a hypothetical protein (UniProt: Q5AVZ4) also 
observed in the (-)AnAA13A $+\mathrm{B}$, was statistically regulated relative to the reference (Additional file 2). This protein showed distant homology to protein annotated as Schizosaccharomyces pombe stress response component (UniProt: P87179.3).

By contrast, the secretomes of (-)AnAA13A and (-)AnAA13A + B revealed 14 and 204 (37 CAZymes) significantly regulated proteins $\left(\log _{2} \mathrm{FC}>2, \mathrm{P} \leq 0.05\right)$, respectively (Fig. 6, Additional file 1: Fig. S7, Additional file 2). Only one of the seven amylolytic hydrolases (GH31, AgdE, UniProt Q5BET9) was amongst the 20 regulated GHs for the (-)AnAA13A + B dataset (Fig. 6, Additional file 2).
Interestingly, this enzyme was downregulated in this dataset, suggestive of a limited access to the oligomers that mediate the transcriptional upregulation. Hypothetical proteins, proteases and a considerable proportion of intracellular proteins were amongst the upregulated proteins, which suggests a distinctly different physiological state of the (-)AnAA13A + B compared to the reference.

\section{Discussion}

The action of LPMOs, their synergy with other hydrolases $[21,22]$ and fungal oxidoreductases $[4,23]$ have been extensively investigated in vitro. LPMOs are proposed to render their substrates more accessible to the

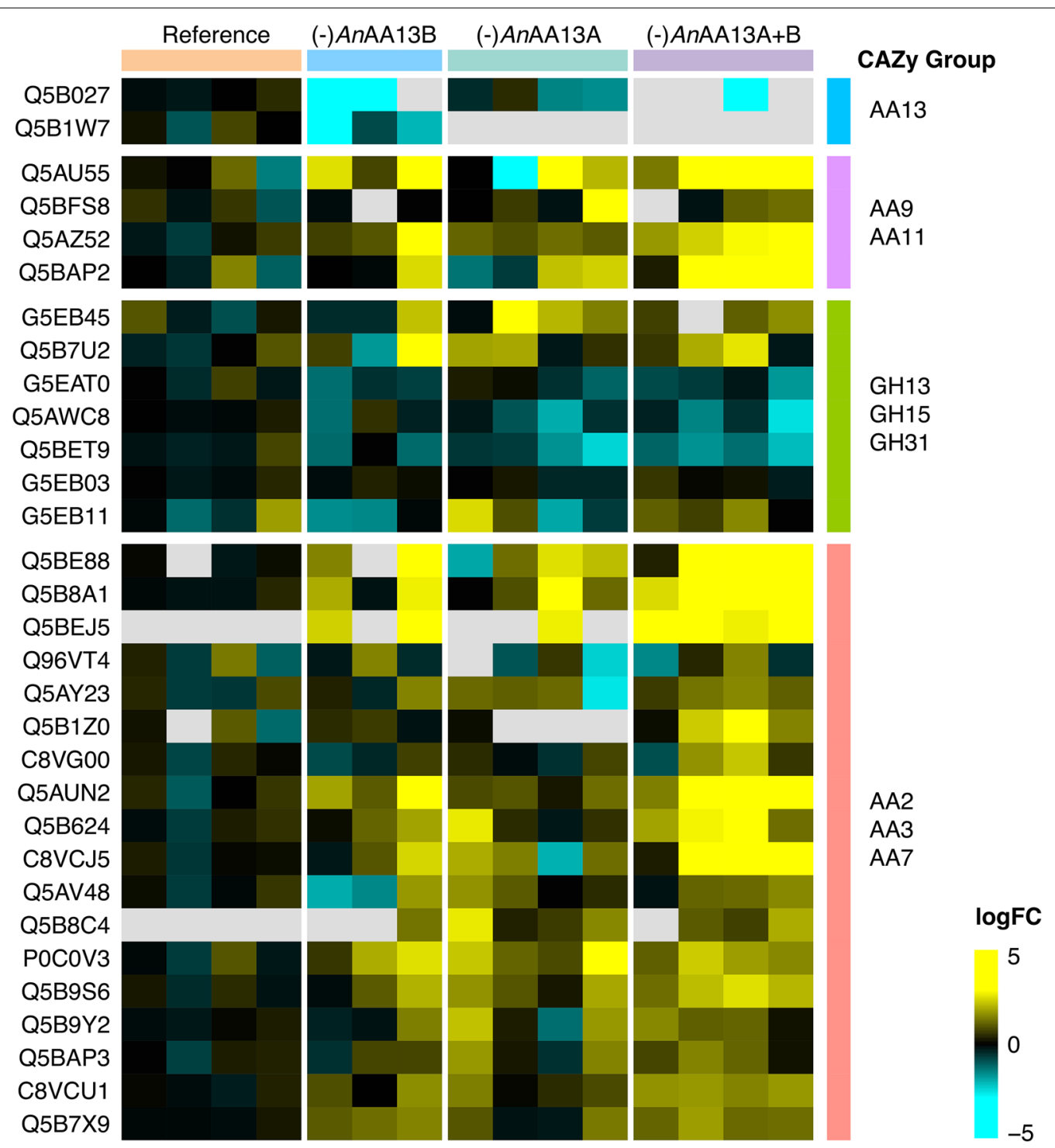

Fig. 6 Heat map comparison of the CAZy families identified in the secretomes of reference and AA13 gene deletion A. nidulans strains at day five of growth on resistant potato starch 
action of hydrolases, as also supported by recent studies showing the role of LPMOs in the disruption of the structure of cellulose fibres [24]. Insight into the actual contribution of distinct LPMOs against more complex backgrounds resembling such secretomes is lacking to date. This study uniquely investigates the impact of AA13 genes on the growth of $A$. nidulans on starches with varying degree of resistance to enzymatic hydrolysis.

The visual phenotypic difference observed for the AA13 gene deletion strains indicated a marked change in growth on resistant potato starch on solid media (Fig. 2). By contrast, these strains grew similarly to the reference on glucose and soluble potato starch, suggesting that the observed AA13-associated phenotypic changes were specific to resistant potato starch with an intact granular structure (Figs. 2, 3, 4, Additional file 1: Fig. S2-S5). An extent of growth on the resistant starch substrate is expected, as all gene deletion strains retain an amylolytic enzyme repertoire that comprises 12 enzymes. Indeed seven of the eight amylolytic hydrolases that have been identified in the secretomes of $A$. nidulans in our previously reported study [13] were detected in the present study (Additional file 2). $\alpha$-Amylolytic and $\alpha$-glucosidase activities were also detected in the AA13 gene deletion strains (Fig. 3). The modest changes in measured activities are supported by the proteomic analyses revealing a slight down-regulation trend of most amylolytic hydrolases (GH13 $\alpha$-amylases, GH31 $\alpha$-glucosidases and GH15 glucoamylases) in the AA13-deficient strains on day 5 (Fig. 6, Additional file 2). The amylolytic hydrolases are likely to preferentially mediate breakdown of less-resistant sites, consistent with the similar growth between the reference and AA13-deficient strains on processed soluble potato starch, where the granular organization has been disrupted (larger extent of $\alpha$-glucan chains are hydrated). By contrast, the depolymerization of the granular form of potato starch is greatly impaired, when one or both of the AA13s is lost.

Our findings highlight a paramount role of the modular starch binding AnAA13A in the degradation of resistant starch, which is supported by: (1) the growth phenotype on solid media (Fig. 2); (2) the microscopic analysis showing a trend of increased amount and size of residual starch granules in (-)AnAA13B, followed by $(-) A n \mathrm{AA} 13 \mathrm{~A}$ and $(-) A n \mathrm{AA} 13 \mathrm{~A}+\mathrm{B}$ as compared to the reference strain (Fig. 3); (3) the secretomic analyses showing only a single significantly regulated protein in the secretomes of $(-) A n \mathrm{AA} 13 \mathrm{~B}$ and the reference strain as opposed to large changes in the secretomes of the (-)AnAA13A and (-)AnAA13A + B strains (Fig. 6, Additional file 2). These observations support the notion that AnAA13A is partly complementing the loss of AnAA13B in the (-)AnAA13B, which does not apply to the same extent for the (-)AnAA13A strain. Only about a fifth of the significantly regulated proteins in the secretomes of the (-)AnAA13A + B strain at day 5 compared to the reference were CAZymes. Thus, the majority were proteins with other functions including proteases and intracellular stress proteins (Additional file 2). This is indicative of large changes in the physiological state of the fungus in the double $(-) A n A A 13 \mathrm{~A}+\mathrm{B}$ strain and/or increased extent of lysis of fungal hyphae due to stress (starvation).

A deleterious effect on the activity of a bacterial modular cellulose-specific LPMO as a result of deleting the appended CBM has been reported [25]. In another fungal cellulose-specific LPMO, the deletion of the CBM also reduced the yield of saccharification, but was not associated with a similar impairment of substrate degradation as above, suggesting that the extent of CBM contribution maybe specific for the enzyme-substrate combination [26]. The abundant secretion of both AA13 LPMOs from $A$. nidulans on wheat, maize and pea starches observed in a previous study [13], demonstrated that AA13s are universally deployed for the degradation of different starches and secondly that these enzymes may exhibit functionally different properties. AnAA13A displayed moderate affinity to the starch mimic $\beta$-cyclodextrin $\left(K_{\mathrm{d}} \approx 40 \mu \mathrm{M}\right)$, which is mediated by the CBM20 starchbinding module [27]. This affinity for $\beta$-cylcodextrin (and starch) is not provided by the active site, as the AnAA13B homologue lacking the CBM20 was not captured by the $\beta$-cyclodextrin affinity column [13]. The active site of AA13 LPMOs has been proposed to recognize the helical conformation of starch in a shallow groove [7, 28], albeit likely with a much lower affinity than the CBM20mediated binding as argued above. A possible advantage of the AnAA13B is that the activity is less localized due to the more dynamic lower affinity binding and smaller size as compared to AnAA13A, which may allow deeper penetration in the starch granule. This feature, maybe more important in cereal starches, especially maize which has a channel structure [19]. A model of inside out degradation mechanism has been proposed for such cereal starches, whereas an exo-corrosion model has been suggested for B-type tuber resistant starches [20], where the starch binding functionality of $A n A A 13 \mathrm{~A}$ is likely to be more important than the smaller size of AnAA13B. Taken altogether, this study demonstrates a crucial role of LPMOs in the degradation of resistant starch, and possibly other semi-crystalline substrates.

\section{Conclusions}

The study provides evidence that the deletion of one or both AA13s reduces the degradation efficiency of granular resistant starch, but not processed solubilized starch. This impact of the AA13 deficiency is less pronounced 
on wheat starch that is more susceptible to degradation by hydrolytic enzymes. This study offers a framework to assess the contribution of distinct LPMOs in fungal secretomes on the utilization of specific semi-crystalline polysaccharides.

\section{Methods}

\section{Carbohydrates and chemicals}

Commercial granular raw resistant potato starch was from Bauckhof (Rosche, Germany), soluble potato starch (S2630) and granular unmodified wheat starch (S5127) were from Sigma-Aldrich (St. Louis, USA). The granular starches for the liquid or solid media growth experiments were not autoclaved to minimize changes of the native starch granule structure, but the soluble starch comprising was autoclaved and used for the growth phenotype assay on plates. The granular starches were thoroughly washed four times with five consecutive volumes of MilliQ water, 70\% ethanol, Milli-Q water and 70\% ethanol at $30{ }^{\circ} \mathrm{C}$ with intermittent centrifugation at 5000xg. The residual ethanol was evaporated in a laminar flow bench and each starch substrate was subsequently resuspended in autoclaved growth media and added to make a homogenous suspension in agar plate before solidification. $p$-Nitrophenyl- $\alpha$-D-glucopyranoside (PNPG) was from Sigma-Aldrich, and insoluble Blue Starch (iBS) was a custom preparation from Pharmacia (Uppsala, Sweden).

\section{Strains and media}

The AnAA13A (Gene locus tag: AN5463 (gene designated aasA in this study), UniProt ID: Q5B1W7) and AnAA13B (Gene locus tag: AN5463 (aasB in this study), UniProt ID: Q5B1W7) starch-specific LPMO encoding genes were deleted in the present work. A. nidulans strain NID1 (argB2, pyrG89, veA, nkuAD) [29] was used as a reference strain for gene deletions. This strain was amenable to efficient genetic manipulation by deficiency for NHEJ (non-homologous end joining) and two auxotrophies, but otherwise resembled wild-type $A$. nidulans strains with regard to ability to utilize complex glycans as carbon source. The $\operatorname{argB}$ and $p y r G$ encode ornithine carbamoyltransferase and orotidine- $5^{\prime}$-phosphate decarboxylase activities, respectively. The $\operatorname{argB2}$ mutant was corrected to functional $\arg B$ in the final mutant strains using CRISPR/Cas9 and the oligonucleotide mediated gene-editing strategy as described recently [30]. The pyrG89 mutation was complemented by the use of the heterologous AfpyrG from Aspergillus fumigatus. This gene, which was extended by its native promoter and terminator and flanked by a direct repeat (DR) of $290 \mathrm{bp}$ [29], was incorporated into the gene-targeting substrates. Genomic DNA (gDNA) from NID1 was isolated using FastDNA SPIN Kit for Soil DNA extraction kit (MP
Biomedicals, USA). All strains employed in this study are listed in Additional file 1: Table S1. Escherichia coli strain $\mathrm{DH} 5 \alpha$ was used as host for plasmid propagations.

Aspergillus nidulans was cultivated on solid and liquid glucose based minimal medium (MM) containing (per litre): $1 \%$ glucose $(\mathrm{w} / \mathrm{v}), 6 \mathrm{~g} \mathrm{NaNO}_{3}, 0.52 \mathrm{~g} \mathrm{KCl}, 0.52 \mathrm{~g}$ $\mathrm{MgSO}_{4} \cdot 7 \mathrm{H}_{2} \mathrm{O}, 1.52 \mathrm{~g} \mathrm{KH}_{2} \mathrm{PO}_{4}$ [18], $0.001 \%$ (w/v) thiamine, and $1 \mathrm{~mL}$ of trace elements [31], 2\% (w/v) agar for solid medium. For the generation of the gene deletion strains, this medium was supplemented with $10 \mathrm{mM}$ uridine (Uri), $10 \mathrm{mM}$ uracil (Ura), and/or $4 \mathrm{mM}$ L-arginine (Arg). Solid plates containing 5-fluoroorotic acid (5-FOA, $1.3 \mathrm{mg} \mathrm{mL}^{-1}$ ) were made from $\mathrm{MM}+\mathrm{Arg}+\mathrm{Uri}+$ Ura for counter-selecting pyrG. The glucose was replaced by $1 \mathrm{M}$ sucrose for transformation media (TM). For protoplasting, $100 \mathrm{~mL}$ of liquid $\mathrm{MM}+\arg +$ Ura + Uri in unbaffled shake flasks were inoculated to a concentration of $1 \times 10^{6}$ conidia $\mathrm{mL}^{-1}$, and incubated at $37{ }^{\circ} \mathrm{C}$ with stirring at $150 \mathrm{rpm}$ for $20 \mathrm{~h}$.

\section{Gene-deletion vector and fungal strain generation}

All PCR products used for cloning were amplified by the proof-reading PfuX7 polymerase [32] on an Agilent sure cycler (Agilent, Santa Clara, CA) using standard PCR programs: $98^{\circ} \mathrm{C}, 2 \mathrm{~min} ; 35$ cycles $\left(98^{\circ} \mathrm{C}, 15 \mathrm{~s} ; 62^{\circ} \mathrm{C}, 30 \mathrm{~s}\right.$; $\left.72{ }^{\circ} \mathrm{C}, 3 \mathrm{~min}\right) ; 72{ }^{\circ} \mathrm{C}, 5 \mathrm{~min}$. The standard reaction volumes were $50 \mu \mathrm{L}$ including $1 \times$ Phusion $^{\circledR} \mathrm{HF}$ Buffer $(\mathrm{New}$ England Biolabs (NEB), USA), $0.2 \mathrm{~mm}$ dNTPs, $0.4 \mu \mathrm{M}$ primers (Integrated DNA Technologies, Belgium), $1 \mathrm{U}$ PfuX7, $<10$ ng of gDNA, 3\% DMSO. PCR products were purified from agarose gels using a GFX purification kit (GE Healthcare, Chicago, USA). All vectors were assembled by USER cloning as described previously [19]. The plasmid's linearization reaction were performed using SwaI (NEB) according to manufacturer's instructions and terminated by heat inactivation $\left(65^{\circ} \mathrm{C}\right.$ for $\left.20 \mathrm{~min}\right)$. Protoplastation and transformation were performed in A. nidulans, respectively, as described before [33, 34]. Transformants were restreaked at least twice, and analysed rigorously by diagnostic tissue-PCR as described elsewhere [34], following the validation scheme, depicted in Additional file 1: Fig. S1.

\section{Growth phenotype assay on solid medium}

The reference $A$. nidulans and the gene deletion strains derived thereof were cultivated on solid $\mathrm{MM}$ at $30{ }^{\circ} \mathrm{C}$ for 5 days. Conidia were gently harvested using ice-cold sterilized $0.9 \%(\mathrm{w} / \mathrm{v}) \mathrm{NaCl}$ solution to a final concentration of $1 \times 10^{8}$ conidia $\mathrm{mL}^{-1}$, which was used to inoculate plates with the same medium containing $1 \%(\mathrm{w} / \mathrm{v})$ or Glc, soluble potato starch or resistant potato starch at three positions. To minimize bacterial contamination of the plates with the washed non-autoclaved resistant potato 
starch, ampicillin $\left(100 \mu \mathrm{g} \mathrm{mL} \mathrm{m}^{-1}\right)$ and chloramphenicol (34 $\mu \mathrm{g} \mathrm{mL} \mathrm{m}^{-1}$ ) were added to the medium together with the starch before solidification. Growth was monitored for up to 9 days.

\section{Confocal laser scanning microscopy (CLSM)}

For the CLSM analysis, $700 \mu \mathrm{L}$ MM containing $1 \%$ agar and either potato or wheat starch $(1 \% \mathrm{w} / \mathrm{v})$ as a sole carbon source, and supplemented with antibiotics as described above, were added as $1.5 \times 4 \mathrm{~cm}$ square to sterilized microscope glass slides. After 30 min solidification, the media were inoculated by adding $10 \mu \mathrm{L}$ of $1 \times 10^{5} \mathrm{~mL}^{-1}$ conidia suspension. Prior to mounting the cover glass onto the incubated slides (with and without inoculum), two drops of $3 \mu \mathrm{L}$ tenfold diluted $0.1 \%(w / v)$ calcofluor white (CFW, Sigma-Aldrich) in Milli-Q were added to the agar with $1 \mathrm{~cm}$ distance between the drops. The microscopy slides were placed in petri dishes stacked in micro-perforated plastic bags at $37{ }^{\circ} \mathrm{C}$ for $20 \mathrm{~h}$. The slides were assembled by a $50 \times 24 \mathrm{~mm}$ cover glass $\# 1$ (Knittel AG, Braunschweig, Germany) and incubated at room temperature for an hour prior microscopy analysis. The assembled slide-samples were mounted up-sidedown on a Leica Microsystems SP8 confocal microscope (Leica, Mannheim, Germany). Images were alternatively captured using a $10 \times \mathrm{HC}$ Plan Fluotar objective, or a $63 \times$ HC N Plan-Apochromat oil immersion objective. Cells stained with CFW were imaged in confocal mode, using $405 \mathrm{~nm}$ excitation and 480-545 $\mathrm{nm}$ emission detection. Transmitted light images were acquired via a non-confocal configuration mode using a $552 \mathrm{~nm}$ laser as illumination. CFW has optimal excitation and emission wavelengths below what were used here, but due to technical limitations, the indicated wavelengths still captured clear images of the fungal cell components.

\section{Growth experiments in liquid medium}

Aspergillus nidulans and the gene-deletion mutants were grown in $250 \mathrm{~mL}$ baffled Erlenmeyer flasks containing $50 \mathrm{~mL} \mathrm{MM}$ containing $1 \%(\mathrm{v} / \mathrm{w})$ resistant potato starch as a carbon source. The MM was supplemented with ampicillin $\left(100 \mu \mathrm{g} \mathrm{mL}^{-1}\right)$ and chloramphenicol $\left(34 \mu \mathrm{g} \mathrm{mL}^{-1}\right)$ to minimize bacterial contamination. Each flask was inoculated to a final concentration of $1 \times 10^{6}$ spores $\mathrm{mL}^{-1}$ and cultivated on a rotary shaker $(150 \mathrm{rpm})$ for 5 days at $30{ }^{\circ} \mathrm{C}$. The growth was performed in four biological replicates.

\section{Biomass, residual starch, amylolytic activities and protein analyses of liquid cultures}

Filtration through a cloth, was used to separate the final fungal biomass (retentate) from the culture suspension.
The wet weight was determined and the biomass was frozen at $-20^{\circ} \mathrm{C}$ until further use. The mannose content of the final fungal biomass was determined by acid hydrolysis. The biomass (from $40 \mathrm{~mL}$ suspension) was thawed, transferred to Teflon-capped pressure tubes, freeze dried, suspended in $1.5 \mathrm{~mL} 72 \%(\mathrm{w} / \mathrm{v}) \mathrm{H}_{2} \mathrm{SO}_{4}$ and incubated at $30{ }^{\circ} \mathrm{C}, 1 \mathrm{~h}$. The samples were immediately cooled down and stored at $5{ }^{\circ} \mathrm{C}$ until further analysis. The mannose content in the samples was quantified using an HPAECPAD system equipped with PA1 column and post-column $\mathrm{NaOH}$ addition as described previously [35].

To separate the residual insoluble resistant potato starch, the culture suspension (filtrate from the above step) was centrifuged at $5000 \times g$ for $30 \mathrm{~min}$. The pelleted starch was washed with twice $96 \%$ ethanol, re-centrifuged as above. The ethanol was removed and the starch dried prior to determining the weight.

For enzyme activity measurements, $500 \mu \mathrm{L}$ culture supernatant was concentrated 50-fold and buffer exchanged to assay buffer (10 mM MES, $0.005 \%$ Triton $\mathrm{X}-100, \mathrm{pH}$ 6.5) using Amicon ${ }^{\circledR}$ Ultra Centrifugal Filters (Merck Millipore, Cork, Ireland).

$\alpha$-Amylase activity of the fungal cultures was assayed toward insoluble Blue Starch (iBS, $6.25 \mathrm{mg} \mathrm{mL}^{-1}$ ) suspended in assay buffer. The reaction mixture $(180 \mu \mathrm{L}$ $\mathrm{iBS}+20 \mu \mathrm{L}$ supernatant) was incubated for $20 \mathrm{~h}$ at $37^{\circ} \mathrm{C}$, stopped by $0.5 \mathrm{M} \mathrm{NaOH}(40 \mu \mathrm{L})$, centrifuged $(20,000 \times g$, $3 \mathrm{~min}$ ), and $200 \mu \mathrm{L}$ supernatant was transferred to a 96 well microtiter plate and the absorbance read at $620 \mathrm{~nm}$. $\alpha$-Glucosidase activity was determined towards $2 \mathrm{mM}$ $p$-nitrophenyl- $\alpha$-D-glucopyranoside (PNPG) in assay buffer. The reaction mixture $(40 \mu \mathrm{L}$ PNPG and $10 \mu \mathrm{L}$ supernatant) was incubated for $20 \mathrm{~h}$ at $37{ }^{\circ} \mathrm{C}$, stopped by adding $1 \mathrm{M} \mathrm{Na}_{2} \mathrm{CO}_{3}(200 \mu \mathrm{L})$, centrifuged $(20,000 \times g$, $3 \mathrm{~min}$ ). Then, $200 \mu \mathrm{L}$ supernatant was transferred to a 96 well microtiter plate and the absorbance read at $410 \mathrm{~nm}$.

The protein concentration was determined using Pierce $^{\mathrm{TM}}$ Rapid Gold BCA Protein Assay Kit (Thermo Fisher Scientific, Waltham, USA) according to the manufacturer's instruction using bovine serum albumin as standard. Amylolytic activities protein concentration were measured in the four biological replicates and presented as the means with standard deviations.

\section{Proteomics}

Samples $(2 \mathrm{~mL})$ from four biological replicate cultures were collected from day 3 to 5 under sterile conditions. The samples were centrifuged $\left(20,000 \times g, 10 \mathrm{~min}, 4{ }^{\circ} \mathrm{C}\right)$ and filtered $(0.2 \mu \mathrm{m}$ regenerated cellulose syringe filters, Thermo Fisher Scientific) to remove mycelia and residual insoluble starch. The filtrate was flash frozen in liquid nitrogen and stored at $-80{ }^{\circ} \mathrm{C}$ until further use. Proteins of the culture filtrates were precipitated 
by addition of acetone to a final concentration of $80 \%$ (v/v), incubation over night at $-20{ }^{\circ} \mathrm{C}$, and centrifugation $(4000 \times g, 10 \mathrm{~min})$ to pellet the precipitated protein. The protein pellets were dissolved in $50 \mu \mathrm{L}$ buffer $(6 \mathrm{M}$ guanidinium hydrochloride, $10 \mathrm{mM}$ Tris(2-carboxyethyl) phosphine hydrochloride, $40 \mathrm{mM}$ 2-chloroacetamide, $50 \mathrm{mM}$ HEPES pH 8.5), boiled $\left(5 \mathrm{~min} 95^{\circ} \mathrm{C}\right)$ and sonicated $\left(5 \times 60 \mathrm{~s}\right.$ at $\left.4{ }^{\circ} \mathrm{C}\right)$ (Bioruptor, Diagenode). For digestion, $45 \mu \mathrm{L}$ sample were diluted 1:3 in digestion buffer (50 mM HEPES, 10\% (v/v) acetonitrile (ACN), pH 8.5) and incubated with $200 \mathrm{ng}$ LysC (MS grade, Wako) at $37{ }^{\circ} \mathrm{C}$ for $3 \mathrm{~h}$. Subsequently, the samples were diluted 1:10 in digestion buffer and further digested with $200 \mathrm{ng}$ trypsin (MS grade, Promega) at $37^{\circ} \mathrm{C}$ for $18 \mathrm{~h}$. The digestion was stopped by addition of $450 \mu \mathrm{L} 2 \%$ trifluoroacetic acid (TFA). Samples were desalted using SOLA $\mu$ HRP plate (Thermo Fisher Scientific) by activation with $200 \mu \mathrm{L}$ methanol and $200 \mu \mathrm{L}$ MS buffer $(80 \% \mathrm{ACN}, 0.1 \%$ formic acid), equilibration $2 \times 200 \mu \mathrm{L} 3 \%$ ACN, 1\% TFA, sample application, $2 \mathrm{x}$ wash with MS buffer A (0.1\% FA) and elution with $2 \times 300 \mu \mathrm{L} \mathrm{40 \%} \mathrm{ACN,} \mathrm{0.1 \%} \mathrm{FA.} \mathrm{Between} \mathrm{each}$ solvent application, the plate was centrifuged $(1500 \mathrm{rpm}$, $1 \mathrm{~min})$. Eluted peptides were dried in an Eppendorf Speedvac $\left(3 \mathrm{~h}\right.$ at $60{ }^{\circ} \mathrm{C}$ ) and reconstituted in $2 \%(\mathrm{v} / \mathrm{v})$ ACN, 1\% (w/v) TFA acid prior to mass spectrometry (MS) analysis. The peptides were loaded on the mass spectrometer by reverse phase chromatography through an inline $15 \mathrm{~cm}$ C18 column (Thermo EasySpray ES804A) connected to a $2 \mathrm{~cm}$ long C18 trap column (164705, Thermo Fisher Scientific) using a Thermo Easy nLC 1200 HPLC system. Peptides were eluted with a mobile phase

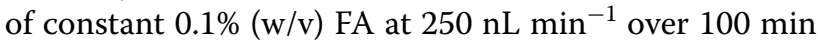
with a five step gradient of buffer B (80\% (v/w) CAN, $0.1 \%$ FA): 0-68 min gradient of $6-23 \%, 68-85 \mathrm{~min} 23-38 \%$, 85-90 min 38-60\%, 90-93 $\min 60-95 \%$, and 93-100 min 95\%. Analysis was performed on a Q-Exactive HF-X instrument (Thermo Fisher Scientific) run in a datadependent manner using a "Top 15" method. Full MS spectra were collected at 60,000 resolution, with an AGC target set to $3 \times 10^{6}$ ions or maximum injection time of $50 \mathrm{~ms}$. Peptides were fragmented via higher-energy collision dissociation (normalized collision energy $=25$ ). The intensity threshold was set to $1 \times 10^{4}$, dynamic exclusion to $60 \mathrm{~s}$ and ions with a charge state $<2,>6$ or unknown species were excluded. MS/MS spectra were acquired at a resolution of 15,000, with an AGC target value of $1 \times 10^{5}$ ions or a maximum injection time of $60 \mathrm{~ms}$. The scan range was limited from 350 to $1850 \mathrm{~m} / z$.

The raw files were analysed using Proteome Discoverer 2.4. Label-free quantitation (LFQ) was enabled in the processing and consensus steps, and spectra were matched against the $A$. nidulans database obtained from Uniprot (UniProt ID: UP000000560).
Dynamic modifications were set as Oxidation (M), Deamidation $(\mathrm{N}, \mathrm{Q})$ and Acetyl on protein N-termini. Cysteine carbamidomethyl was set as a static modification. All results were filtered to a $1 \%$ FDR, and protein quantitation done using the built-in Minora Feature Detector.

The normalized abundance values for each sample were then $\log _{2}$ transformed and used for further hypothesis testing. A linear modelling approach (LIMMA v3.40.6) was applied using the $\mathrm{R}$ statistical computing environment (v3.6.1). After a principal component analysis of the protein abundances from day 3 and day 5 samples, the largest source of variability was observed to be related to the sampling day, and that day 3 samples showed little effects due to the strain background. For this reason, only day 5 samples were used in the statistical model. A linear model was applied where log2-abundance values were used to estimate the parameters for each strain background and comparisons (contrasts) were made for each $\mathrm{KO}$ strain relative to the reference strain. Empirical Bayes statistics were used to estimate false discovery rates $(q)$ using the Benjamini-Hochberg method for each comparison. The difference in the group means is represented by the $\log _{2}$-fold change $(\log$ FC) from the reference. The mass spectrometry proteomics data have been deposited to the ProteomeXchange Consortium via the PRIDE [21] repository under the entry PXD020101.

\section{Supplementary information}

Supplementary information accompanies this paper at https://doi. org/10.1186/s13068-020-01775-z.

Additional file 1: Table S1. List of strains used in the study. Table S2. Primers for generation and validation of the A. nidulans mutant strains. Fig. S1. Gene targeting and mutant-strain validation. Fig. S2. Growth of reference and AA13 gene deletion strains on glucose. Fig. S3. Growth of reference and AA13 gene deletion strains on soluble potato starch. Fig. S4. Growth of reference and AA13 gene deletion strains on resistant potato starch. Fig. S5. Biomass analysis of reference and AA13 gene deletion strains on resistant potato starch at day 5. Fig. S6. Principal component analysis of the proteomic data sets during growth on resistant starch. Fig. S7. Venn diagram of the significantly upregulated proteins during growth on resistant potato starch.

Additional file 2: Label-free Proteomics.

\section{Abbreviations}

AA: Auxiliary activity enzyme; CAZy: Carbohydrate-active enzyme; CBM: Carbohydrate-binding module; CFW: Calcofluor white; CLSM: Confocal laser scanning microscopy (CLSM) analysis; KOs: Knock-outs; LPMO: Lytic polysaccharide monooxygenase; MM: Minimal media; NHEJ: Non-homologous end joining; SS: Soluble potato starch; RS: Resistant potato starch.

\section{Acknowledgements}

Mikkel Sloth Rasmussen is thanked for technical assistance with the fungal growth.

\section{Authors' contributions}

$\mathrm{MHM}$ and MAH conceived the research and designed the experiments. JBH designed the genetic engineering experiments, which were performed and 
validated by JBH and MHM. MHM performed the initial growth experiments. MLL performed the solid and liquid media growth experiments, activity, protein, residual starch measurements for the revised manuscript. MHM, JBH, and CS performed the microscopic analysis. JH performed the mannose biomass analysis. MLL, MAH and CTW made the figures for the revised manuscript. ES and MWN performed the proteomics experiments and the initial search for protein identification. CTW performed the proteomics data analysis and hypothesis testing. MHM, JBH and MAH wrote the first draft of the manuscript. All authors read and approved the final manuscript.

\section{Funding}

This study was supported by a Novo Nordisk foundation Post-doc grant within the Biotechnology-based Synthesis and Production Research program to MHM (NNF17OC0025642). Additional funding was from an interdisciplinary synergy Grant (NNF180C0034918).

\section{Availability of data and materials}

The A. nidulans gene-deletion strains are available at DTU Bioengineering. The MS proteomics data have been deposited to the ProteomeXchange Consortium with the dataset identifier PXD020101.

\section{Ethics approval and consent to participate}

Not applicable.

\section{Consent for publication}

All authors have seen the manuscript and consent for publication.

\section{Competing interests}

The authors declare no competing interest.

Received: 19 February 2020 Accepted: 24 July 2020

Published online: 05 August 2020

\section{References}

1. Cragg SM, Beckham GT, Bruce NC, Bugg TDH, Distel DL, Dupree P, et al. Lignocellulose degradation mechanisms across the Tree of Life. Curr Opin Chem Biol. 2015;2015(29):108-19.

2. Bouws $\mathrm{H}$, Wattenberg A, Zorn H. Fungal secretomes - Nature's toolbox for white biotechnology. Appl Microbiol Biotechnol. 2008;80:381-8.

3. Vaaje-Kolstad G, Westereng B, Horn SJ, Liu Z, Zhai H, Sørlie M, et al. An oxidative enzyme boosting the enzymatic conversion of recalcitrant polysaccharides. Science. 2010;330(6001):219-22.

4. Eijsink VGH, Ludwicka K, Felice AKG, Preims M, Ludwig R, BresImayr E, et al. Extracellular electron transfer systems fuel cellulose oxidative degradation. Science. 2016;352:1098-101.

5. Bissaro B, Røhr ÅK, Müller G, Chylenski P, Skaugen M, Forsberg Z, et al. Oxidative cleavage of polysaccharides by monocopper enzymes depends on $\mathrm{H}_{2} \mathrm{O}_{2}$. Nat Chem Biol. 2017;13:1123-8.

6. Frommhagen M, Sforza S, Westphal AH, Visser J, Hinz SWA, Koetsier MJ, et al. Discovery of the combined oxidative cleavage of plant xylan and cellulose by a new fungal polysaccharide monooxygenase. Biotechnol Biofuels. 2015;8(1):101.

7. Lo Leggio L, Simmons TJ, Poulsen JCN, Frandsen KEH, Hemsworth GR, Stringer MA, et al. Structure and boosting activity of a starch-degrading lytic polysaccharide monooxygenase. Nat Commun. 2015;6:5961.

8. Forsberg Z, Vaaje-kolstad G, Westereng B, Bunsæ AC, Stenstrøm Y, Mackenzie $A$, et al. Cleavage of cellulose by a cbm33 protein. Protein Sci. 2011;20:1479-83.

9. Agger JW, Isaksen T, Várnai A, Vidal-Melgosa S, Willats WGT, Ludwig $R$, et al. Discovery of LPMO activity on hemicelluloses shows the importance of oxidative processes in plant cell wall degradation. Proc Natl Acad Sci USA. 2014;11(17):6287-92.

10. Beeson WT, Vu VV, Span EA, Phillips CM, Marletta MA. Cellulose degradation by polysaccharide monooxygenases. Annu Rev Biochem. 2015;84:923-46.

11. Coutinho PM, Andersen MR, Kolenova K, vanKuyk PA, Benoit I, Gruben $\mathrm{BS}$, et al. Post-genomic insights into the plant polysaccharide degradation potential of Aspergillus nidulans and comparison to Aspergillus niger and Aspergillus oryzae. Fungal Genet Biol. 2009;46(Suppl):1.
12. Pérez $\mathrm{S}$, Bertoft $\mathrm{E}$. The molecular structures of starch components and their contribution to the architecture of starch granules: a comprehensive review. Starch/Staerke. 2010;62:389-420.

13. Nekiunaite L, Arntzen M, Svensson B, Vaaje-Kolstad G, Abou Hachem M. Lytic polysaccharide monooxygenases and other oxidative enzymes are abundantly secreted by Aspergillus nidulans grown on different starches. Biotechnol Biofuels. 2016;9:1-16.

14. Streb S, Zeeman SC. Starch metabolism in arabidopsis. 2012;10:e0160.

15. Hizukuri S, Takeda Y, Yasuda M, Suzuki A. Multi-branched nature of amylose and the action of debranching enzymes. Carbohydr Res. 1981;94:205-13.

16. Levasseur A, Drula E, Lombard V, Coutinho PM, Henrissat B. Expansion of the enzymatic repertoire of the CAZy database to integrate auxiliary redox enzymes. Biotechnol Biofuels. 2013;6:41.

17. Christiansen C, Abou Hachem M, Janeček Š, Viksø-Nielsen A, Blennow A, Svensson B. The carbohydrate-binding module family 20-diversity, structure, and function. FEBS J. 2009;276:5006-29.

18. Kaminskyj SGW. Fundamentals of growth, storage, genetics and microscopy of Aspergillus nidulans. Fungal Genet Rep. 2001;48:25-31.

19. Hansen BG, Salomonsen B, Nielsen MT, Nielsen JB, Hansen NB, Nielsen $\mathrm{KF}$, et al. Versatile enzyme expression and characterization system for Aspergillus nidulans, with the Penicillium brevicompactum polyketide synthase gene from the mycophenolic acid gene cluster as a test case. Appl Environ Microbiol. 2011;77:3044-51.

20. Gow NAR, Latge J-P, Munro CA. The fungal cell wall: structure, biosynthesis, and function. Microbiol Spectr. 2017;5:1-25.

21. Forsberg Z, Sørlie M, Petrović D, Courtade G, Aachmann FL, VaajeKolstad G, et al. Polysaccharide degradation by lytic polysaccharide monooxygenases. Curr Opin Struct Biol. 2019;59:54-64.

22. Tandrup T, Frandsen KEH, Johansen KS, Berrin JG, Leggio L. Recent insights into lytic polysaccharide monooxygenases (LPMOs). Biochem Soc Trans. 2018;46(6):1431-47.

23. Garajova S, Mathieu Y, Beccia MR, Bennati-Granier C, Biaso F, Fanuel $M$, et al. Single-domain flavoenzymes trigger lytic polysaccharide monooxygenases for oxidative degradation of cellulose. Sci Rep. 2016;6:28276.

24. Villares A, Moreau C, Bennati-Granier C, Garajova S, Foucat L, Falourd X, et al. Lytic polysaccharide monooxygenases disrupt the cellulose fibers structure. Sci Rep. 2017;7:40262.

25. Crouch LI, Labourel A, Walton PH, Davies GJ, Gilbert HJ. The contribution of non-catalytic carbohydrate binding modules to the activity of lytic polysaccharide monooxygenases. J Biol Chem. 2016;291(14):7439-49.

26. Chalak A, Villares A, Moreau C, Haon M, Grisel S, D'Orlando A, et al. Influence of the carbohydrate-binding module on the activity of a fungal AA9 lytic polysaccharide monooxygenase on cellulosic substrates. Biotechnol Biofuels. 2019;12(1):206.

27. Nekiunaite L, Isaksen T, Vaaje-Kolstad G, Abou Hachem M. Fungal lytic polysaccharide monooxygenases bind starch and $\beta$-cyclodextrin similarly to amylolytic hydrolases. FEBS Lett. 2016;590:2737-47.

28. Vu VV, Hangasky JA, Detomasi TC, Henry SJW, Ngo ST, Span EA, et al. Substrate selectivity in starch polysaccharide monooxygenases. J Biol Chem. 2019:294:12157-66.

29. Nielsen JB, Nielsen ML, Mortensen UH. Transient disruption of nonhomologous end-joining facilitates targeted genome manipulations in the filamentous fungus Aspergillus nidulans. Fungal Genet Biol. 2008;45(3):165-70.

30. Nødvig CS, Hoof JB, Kogle ME, Jarczynska ZD, Lehmbeck J, Klitgaard DK, et al. Efficient oligo nucleotide mediated CRISPR-Cas9 gene editing in Aspergilli. Fungal Genet Biol. Academic Press Inc.; 2018;115:78-89.

31. Cove DJ. The induction and repression of nitrate reductase in the fungus Aspergillus nidulans. Biochim Biophys Acta. 1966;113:51-6.

32. Nørholm MHH. A mutant Pfu DNA polymerase designed for advanced uracil-excision DNA engineering. BMC Biotechnol. 2010;10:21.

33. Nielsen ML, Albertsen L, Lettier G, Nielsen JB, Mortensen UH. Efficient PCR-based gene targeting with a recyclable marker for Aspergillus nidulans. Fungal Genet Biol. 2006;43(1):54-64.

34. Nødvig CS, Nielsen JB, Kogle ME, Mortensen UH. A CRISPR-Cas9 system for genetic engineering of filamentous fungi. PLOS ONE. 2015;10(7):e0133085. 
35. Zeuner B, Muschiol J, Holck J, Lezyk M, Gedde MR, Jers C, et al. Substrate specificity and transfucosylation activity of $\mathrm{GH} 29$ a-L-fucosidases for enzymatic production of human milk oligosaccharides. New Biotechnol. 2018:41:34-45.

\section{Publisher's Note}

Springer Nature remains neutral with regard to jurisdictional claims in published maps and institutional affiliations.
Ready to submit your research? Choose BMC and benefit from:

- fast, convenient online submission

- thorough peer review by experienced researchers in your field

- rapid publication on acceptance

- support for research data, including large and complex data types

- gold Open Access which fosters wider collaboration and increased citations

- maximum visibility for your research: over 100M website views per year

At BMC, research is always in progress.

Learn more biomedcentral.com/submissions 